\title{
Inductive seismo-electromagnetic effect in relation to seismogenic ULF emission
}

\author{
O. Molchanov ${ }^{1}$, A. Kulchitsky ${ }^{2,3}$, and M. Hayakawa ${ }^{2}$ \\ ${ }^{1}$ EORC, NASDA, Roppongi 1-9-9, Minato-ku, Tokyo 106-0032, Japan \\ ${ }^{2}$ University of Electro-Communications, Chofu 1-5-1, Tokyo 182-8585, Japan \\ ${ }^{3}$ Moscow Lomonosov State University, Vorobievy gory, 119899, Moscow, Russia
}

Received: 21 May 2001 - Accepted: 25 June 2001

\begin{abstract}
During the seismic wave propagation through the crust, the electromagnetic pulse can originate due to MHD conversion in this conductive medium. On the assumption of simple models of seismic wave excitation and attenuation, the problem is reduced to the analysis of a diffusionlike equation for a vector potential function. In this way, we need to change the classical gauge condition. A semianalytical form of the solution is obtained in a case with constant ground conductivity. Dependencies of the electric and magnetic field components and the pulse duration on distance and crust conductivity have been computed in detail. The results could be useful for the explanation of electromagnetic signals related to coseismic, foreshock and aftershock activity.
\end{abstract}

\section{Introduction}

ULF (ultra-low frequency, $f=0.003-10 \mathrm{~Hz}$ ) electromagnetic emissions have been observed prior to the occurrence of earthquakes in the USA, Russia, and Japan (see references in Hayakawa, 1999). It is rather difficult to find theoretical mechanisms of this phenomenon. Fenoglio et al. (1994) proposed a model of ruptured isolated reservoirs, resulting in the electro-kinetic (EK) generation of a transient magnetic field. However, they neglected the compensating conduction current inside the path of the EK current and they oversimplified the problem. Therefore, EK generation can be used for the interpretation of so-called seismo-electric signals (SES), but not for ULF magnetic emission (Molchanov, 1999).

A mechanism of ULF electromagnetic field generation based on the ensemble crack opening (microfracturing) was considered by Molchanov and Hayakawa (1995). Creation and relaxation of charges at the walls of opening cracks in the earthquake hypocenter was proposed as a possible reason for electromagnetic noise prior to the earthquake. The

Correspondence to: A. Kulchitsky

(antonk@whistler.ee.uec.ac.jp) other approach was suggested by Surkov (1999), Surkov et al. (2000), and Molchanov et al. (2001). Their theory is based on the same assumption of microfracturing, but the electromagnetic perturbation is caused by an MHD (inductive) effect from a propagating seismic wave. An inductive seismomagnetic effect from a solitary seismic wave was assumed in relation to the observation of so-called magnetic forerunners or coseismic magnetic perturbations (Eleman, 1965; Belov et al., 1974). Recently, the coseismic electric signals were also observed (Takeuchi et al., 1997; Nagao et al., 2000).

The next problem is the penetration of the electromagnetic fields through a dissipative ground medium. The conventional way is to consider static fields. For example, Fenoglio et al. (1994) applied the Biot-Savart law for the estimation of magnetic variations from the opening fracture. But Kawate et al. (1998) found a clear difference between the results of modeling on the suppositions of Biot-Savart law and full-wave procedure. However, they have assumed a quasistationary source. The same approach of quasi-stationarity is conventional for magneto-telluric (MT) or telluric calculations (e.g. Ward and Hohmann, 1988), but it is not valid for our case of a pulsing source. In a low-frequency (LF) domain after neglecting the displacement currents (it is valid for a ground medium if $f<1 \mathrm{MHz}$ ), Maxwell equations can be reduced to the well-known equations of electromagnetic diffusion (e.g. Hohmann, 1988):

$$
\begin{aligned}
& \frac{1}{D} \frac{\partial \boldsymbol{H}}{\partial t}-\nabla^{2} \boldsymbol{H}=\nabla \times \boldsymbol{j}_{s}+\nabla \sigma \times \boldsymbol{E}, \quad D=\frac{1}{\mu \sigma} \\
& \frac{1}{D} \frac{\partial \boldsymbol{E}}{\partial t}-\nabla^{2} \boldsymbol{E}=\nabla\left(\frac{1}{\sigma} \nabla \cdot \boldsymbol{j}_{s}\right) \\
&+\nabla\left(\boldsymbol{E} \cdot \frac{\nabla \sigma}{\sigma}\right)-\mu \frac{\partial \boldsymbol{j}_{s}}{\partial t}
\end{aligned}
$$

where $\boldsymbol{E}, \boldsymbol{H}, \boldsymbol{j}_{s}$ are the vectors of electric and magnetic fields and source current density, $\sigma, \mu$ are conductivity and magnetic permeability, and $D=(\sigma \mu)^{-1}$ is the coefficient of electromagnetic diffusion. 


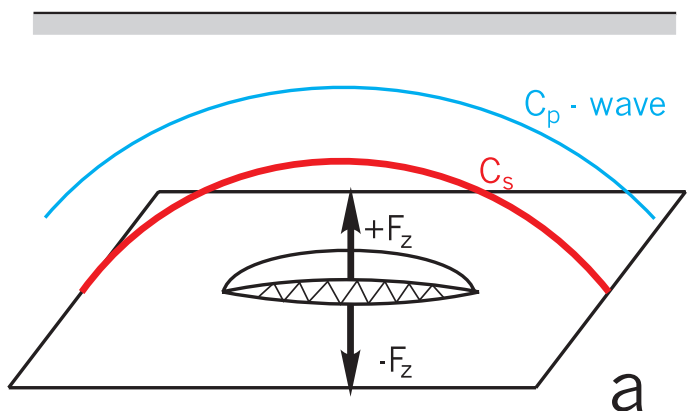

Fig. 1. Scheme of tension (a) shear (b) cracks.

These equations are rather popular in MHD theory and could be used for the special assumptions on parameters (e.g. frozen electric field). But in our case, they lead to great complications due to the imposing vectorial operators on the source current. For example, even by using the assumption of constant conductivity, Surkov et al. (2000) have succeeded in obtaining only asymptotic behaviour of the solutions, which cannot be checked in a static limit and cannot be applied for comparison with observational data. The numerical solution of these equations are sometimes used in an MT study in order to analyze the transient fields (Hohmann, 1988; Pellerin and Hohmann, 1995). But such 4-D computations usually require a lot of time and also cannot be checked in dependence on the task parameters. We found that this is the best way to use the classical double-potential method but with special modification of the gauge condition.

\section{Basic equations}

Vectorial potential $\boldsymbol{A}$ and scalar potential $\varphi$ are introduced in the usual manner:

$\boldsymbol{H}=\boldsymbol{\nabla} \times \boldsymbol{A} \quad \boldsymbol{E}=-\mu \frac{\partial \boldsymbol{A}}{\partial t}-\nabla \varphi$.

These potentials are not completely independent from each other. Their connection is determined by the so-called gauge; its classical (Lorentz) form is the following (Landau and Liftshits, 1992):

$\boldsymbol{\nabla} \cdot \boldsymbol{A}+\varepsilon \frac{\partial \varphi}{\partial t}=0 \quad$ or $\quad \boldsymbol{\nabla} \cdot \boldsymbol{A}=0$.

The last relationship is conventional in LF and static approximation. But this gauge cannot give separated second order equations for the potentials in the case of $\sigma \neq 0$. On the other hand, it is usual (e.g. in plasma physics) to include the conductivity influence through the effective, complex permittivity coefficient $\varepsilon^{\prime}=\varepsilon(1+i \sigma / \omega \varepsilon)$. It identified how the classic gauge could be modified. Remembering that operator $\partial / \partial t \simeq-i \omega$, we can rewrite this gauge as follows:

$$
\begin{gathered}
\nabla \cdot \boldsymbol{A}+\varepsilon^{\prime} \frac{\partial \varphi}{\partial t}=\nabla \cdot \boldsymbol{A}+\varepsilon\left(1+\frac{i \sigma}{\omega \varepsilon}\right)(-i \omega \varphi) \simeq \\
\nabla \cdot \boldsymbol{A}+\varepsilon \frac{\partial \varphi}{\partial t}+\sigma \varphi=0
\end{gathered}
$$

and introduce a new gauge for the conductive medium in LF approximation:

$\nabla \cdot \boldsymbol{A}+\sigma \varphi=$ const.

Using this gauge, the equations for vectorial and scalar potentials are written as follows:

$\frac{1}{D} \frac{\partial \boldsymbol{A}}{\partial t}-\nabla^{2} \boldsymbol{A}+(\nabla \sigma / \sigma) \nabla \cdot \boldsymbol{A}=\boldsymbol{j}_{s}$

$\nabla(\sigma \varphi)=-\nabla(\nabla \cdot A)$.

Of course, these equations are more convenient for computations than Eqs. $(1,2)$ and Eq. (7) clearly represents the combination of diffusion and convection processes. But in the case of a homogeneous conductive medium, the situation is reduced to a symmetrical view of simple diffusion equations:

$\frac{1}{D} \frac{\partial \boldsymbol{A}}{\partial t}-\nabla^{2} \boldsymbol{A}=\boldsymbol{j}_{s}$

$\frac{1}{D} \frac{\partial \varphi}{\partial t}-\nabla^{2} \varphi=-\nabla \cdot \boldsymbol{j}_{s} / \sigma$

where $D=(\sigma \mu)^{-1}$ is the coefficient of electromagnetic diffusion.

The electric field induced in the conductor moving with velocity $\boldsymbol{v}$ under the permanent magnetic field $\boldsymbol{H}_{0}$ is known:

$\boldsymbol{E}^{\prime}=\mu \boldsymbol{v} \times \boldsymbol{H}_{0}$

assuming $\boldsymbol{H} \ll \boldsymbol{H}_{0}$. In our case, $\boldsymbol{v}=\partial \boldsymbol{u} / \partial t$, where $\boldsymbol{u}$ is a vector of ground displacement produced by a seismic wave, and the resultant source current is given by,

$\boldsymbol{j}_{s}=\sigma \boldsymbol{E}^{\prime}=\frac{1}{D} \frac{\partial \boldsymbol{u}}{\partial t} \times \boldsymbol{H}_{0}$

\section{Model of seismic perturbation}

In general, the seismic source is characterized by seismic moment density tensor (Aki and Richards, 1980). However, for the description of far-distance displacement (distance $r \gg L_{S}$, the last is the source dimension), the seismic moment $M$, which is proportional to the faulting area $A_{s} \sim L_{s}^{2}$ and slip [ $u$ ], is conventionally used. It is equivalent 


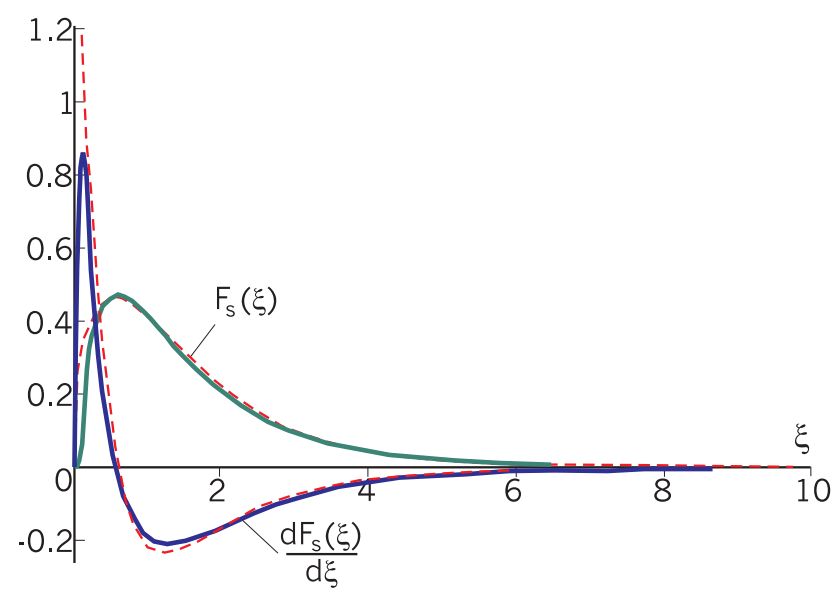

Fig. 2. Function $F_{S}(\xi)$ and $d F_{S} / d \xi$ (Eq. (20)). Solid line is the approximation.

to the supposition of the point source and a couple of body forces. There are two limiting cases in such a way (Fig. 1). The first is a case of expansion or tension crack when a force direction (direction of strike) is perpendicular to the faulting plane (Fig. 1a). The second (Fig. 1b) is a case of shear dislocation when strike and slip are in the faulting plane. Two waves are propagating from the seismic source with velocity $C_{p}=\sqrt{(l+2 m) / \rho}$ (primary wave P) and $C_{s}=\sqrt{m / \rho}$, where $m, l$ are Lame's coefficients and $\rho$ is the ground density. By neglecting attenuation, they create the following displacements (Aki and Richards, 1980):

a) in the case of a tension crack, $M_{t}=l A_{s}\left[u_{z}\right]$,

$$
\boldsymbol{u}_{t}^{i}=\frac{\boldsymbol{a}_{t}^{i}}{4 \pi \rho C_{i}^{3} r} \frac{\partial M_{t}\left(t-r / C_{i}\right)}{\partial t}
$$

where $i=P, S$. In the spherical coordinate system with unity vectors $\boldsymbol{e}_{r}, \boldsymbol{e}_{\theta}, \boldsymbol{e}_{\phi}$ (axis $\theta=0$ is perpendicular to the faulting plane, and axis $\phi=0$ is the strike direction), the polarization vectors are the following:

$$
\boldsymbol{a}_{t}^{P}=\left(1+2 m \cos ^{2} \theta / l\right) \boldsymbol{e}_{r}, \boldsymbol{a}_{t}^{S}=-m \sin 2 \theta \boldsymbol{e}_{\theta} / l
$$

b) in the case of a shear crack, $M_{s h}=m A_{s}\left[u_{x}\right]$

$$
\boldsymbol{u}_{s h}^{i}=\frac{\boldsymbol{a}_{s h}^{i}}{4 \pi C_{i}^{3} r} \frac{\partial M_{s h}\left(t-r / C_{i}\right)}{\partial t}
$$

and

$$
\begin{aligned}
\boldsymbol{a}_{s h}^{P} & =\sin 2 \theta \cos \left(\phi-\phi_{s}\right) \boldsymbol{e}_{r} \\
\boldsymbol{a}_{s h}^{S} & =\cos 2 \theta \cos \left(\phi-\phi_{s}\right) \boldsymbol{e}_{\theta} \\
& =\cos \theta \sin \left(\phi-\phi_{s}\right) \boldsymbol{e}_{\phi}
\end{aligned}
$$

where $\phi_{s}$ is the angle between the directions of strike and slip (see Fig. 1b).

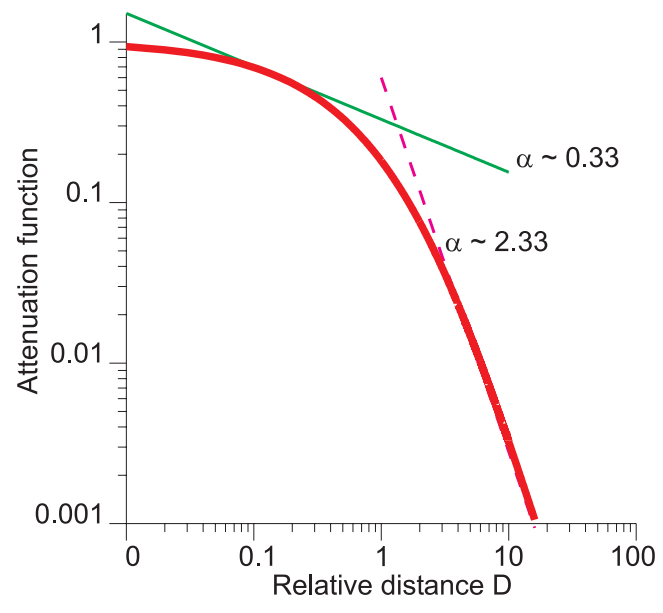

Fig. 3. Attenuation function for the relative distance $r / R_{a}$. It can be represented by the dependence $\left(r / R_{a}\right)^{-\alpha}$, where $\alpha \approx 2.33$ for $r \geq 3 R_{a}$ and averaged $\alpha \approx 0.33$ for the values $r \leq R_{a}$.

Lame's coefficients are related with rigidity $K_{t}=2 m$ and the Poisson ratio $p: l=K_{t} p /(1-2 p)$. As usual in theory, we assume $p \sim 0.33$, so $l \sim K_{t}$ and $C_{p} \sim 2 C_{s}$. Furthermore, we simplify the problem supposing:

- $\boldsymbol{H}_{0}$ is parallel to the axis $\theta=0$

$-\phi_{s}=0$

Eqs. $(13,15)$ include the dependence of $\partial M / \partial t$ on retard time $t-r / C_{i} \geq 0$.

The temporal evolution of $M(r, t)$ can be expressed as follows:

$\left.M\right|_{t \geq 0}=\int_{0}^{\infty} M\left(\omega / \omega_{c}\right) e^{-i \omega t} d \omega$

where $\omega_{c}$ is a "corner" frequency of the seismic spectrum and $\omega_{c} \tau=q_{0} \approx 2$ (e.g. Brune et al., 1979), $\tau \approx L_{s} / C_{R}$ is the duration of the slip, and $C_{R}$ is the rupture velocity. $M\left(\omega / \omega_{c}\right)$ is proportional to $\omega^{-3}$ in the high frequency $\left(\omega \gg \omega_{c}\right)$ part of the spectrum (Aki and Richards, 1980, Scholz, 1990). So the convenient representation is the following:

$M\left(\omega / \omega_{c}\right)=\left(M_{0} / \omega_{c}\right) /\left[1+\left(\omega / \omega_{c}\right)^{2}\right]^{3 / 2}$

where $M_{0}=K_{t} A_{s}\left[u_{z}^{0}\right]$ for a tension crack or $M_{0}=$ $K_{t} A_{s}\left[u_{x}\right] / 2$ for a shear crack. Hence, by neglecting attenuation, we obtain

$\left.\frac{\partial M}{\partial t}\right|_{t \geq r / C_{i}}=M_{0} \omega_{c} F_{S}(\xi)$

where $\xi=\omega_{c}\left(t-r / C_{i}\right)$, and for the displacement, we have

$F_{S}(\xi)=\int_{0}^{\infty} \frac{x \sin (x \xi) d x}{\left(1+x^{2}\right)^{3 / 2}} \approx 20 \pi \xi^{2} \exp (-5 \sqrt{\xi})$ 


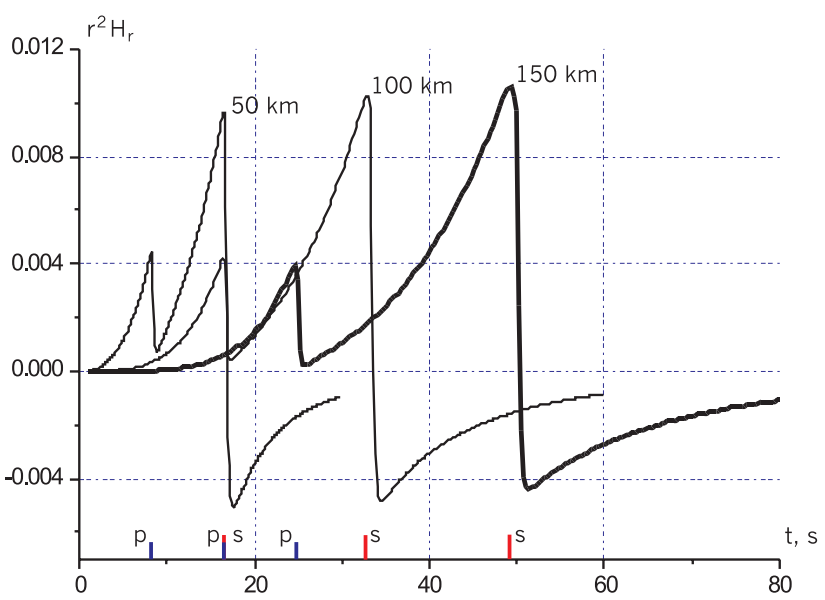

Fig. 4. Calculation $r^{2} H_{r}(t)$ for $r=50 \mathrm{~km}, r=100 \mathrm{~km}, r=$ $150 \mathrm{~km} . \theta=15^{\circ}$. Figure shows that $H_{r} \sim 1 / r^{2}$. Time of primary $(p)$ and secondary $(s)$ seismic wave are highlighted.

The last expression is a useful approximation that is demonstrated in Fig. 2. For this approximation, $d F_{s} / d \xi$ (velocity) is limited for all $\xi \in[0,+\infty)$. Furthermore, the condition of summary displacement $\int_{0}^{+\infty} d F_{S}(\xi)=0$ is true.

As a result, the induced source currents are the following:

$$
\begin{aligned}
\boldsymbol{j}_{t}= & B_{t}\left\{f^{P}(r, t) \sin \theta\left(1+\cos ^{2} \theta\right)\right. \\
& \left.+8 f^{S}(r, t) \sin \theta \cos ^{2} \theta\right\} \boldsymbol{e}_{\phi}
\end{aligned}
$$

$$
\begin{aligned}
& \boldsymbol{j}_{s h}=B_{s h}\left\{f^{P} \sin \theta \sin 2 \theta \cos \phi \boldsymbol{e}_{\phi}+8 f^{S} \cos \theta\right. \\
& \left.\left[\sin \theta \sin \phi \boldsymbol{e}_{r}+\cos \theta \sin \phi \boldsymbol{e}_{\theta}+\cos 2 \theta \cos \phi \boldsymbol{e}_{\phi}\right]\right\}
\end{aligned}
$$

where

$$
B_{t}=H_{0} A_{s} C_{R}\left[u_{z}^{0}\right] /\left(8 \pi D C_{p} L_{s}^{2}\right) \approx C H_{0}\left[u_{z}^{0}\right] /(16 D)
$$

assuming

$C \approx C_{R} \approx C_{s} \approx C_{p} / 2, \quad B_{s h} \approx C H_{0}\left[u_{x}^{0}\right] /(32 D)$

and

$f^{i}=\left(T_{a}^{i} / r\right) \frac{\partial F_{s}}{\partial \xi}, \quad \xi=2\left(t-t_{s}^{i}\right) / \tau \geq 0$

$t_{s}^{i}=r / C_{i}, i=P, S$. Here, $T_{a}^{i}$ is an attenuation function. It depends on the spectrum of seismic pulse (see Molchanov et al., 2001):

$$
\begin{aligned}
T_{a}^{i}= & \left\{\int \omega^{4} M^{2}\left(\omega / \omega_{c}\right) \exp \left(-2 \omega r / \omega_{c} R_{a}^{i}\right)\right. \\
& \left.d \omega / \int \omega^{4} M^{2}\left(\omega / \omega_{c}\right) d \omega\right\}^{1 / 2}
\end{aligned}
$$

where $R_{a}^{i}=2 Q C_{i} / \omega_{c} \approx Q L_{s} C_{i} / C$ is an attenuation distance, $Q$ is a seismic quality ( $Q \approx 100$ ). Assuming (18), we have the following expression for $T_{a}$ :

$$
\begin{aligned}
& T_{a}\left(r / R_{a}\right) \\
& \quad=\frac{4}{\sqrt{3 \pi}}\left\{\int_{0}^{\infty} x^{4} \exp \left(-2 x r / R_{a}\right) d x /\left(1+x^{2}\right)^{3}\right\}^{1 / 2}
\end{aligned}
$$

where $R_{a}^{i} \approx Q L_{s} C_{i} / C$. The dependence of $T_{a}\left(r / R_{a}\right)$ is given in Fig. 3 .

\section{Solution for a tension crack}

In this case, $\boldsymbol{A}=A \boldsymbol{e}_{\phi}, \nabla \cdot \boldsymbol{A}=0, \varphi=0, E_{\phi}=-\mu \partial A / \partial t$, $H_{r}=(1 / r \sin \theta) \partial(\sin \theta A) / \partial \theta, H_{\theta}=-(1 / r) \partial(r A) / \partial r$, $H_{\phi}=E_{r}=E_{\theta}=0$ and the basic equation (9) is reduced to the following:

$$
\begin{aligned}
\frac{1}{D} \frac{\partial A^{i}}{\partial t} & -\frac{1}{r^{2}} \frac{\partial}{\partial r}\left(r^{2} \frac{\partial A^{i}}{\partial r}\right)-\frac{1}{r^{2}} \frac{\partial}{\partial \theta}\left(\frac{1}{\sin \theta} \frac{\partial A^{i} \sin \theta}{\partial \theta}\right) \\
& =B^{i} f^{i}(t, r) \Phi^{i}(\theta)
\end{aligned}
$$

where the meaning of $\Phi^{i}(\theta)$ and $B^{i}$ is evident from Eq. (21). Index $i$ will be omitted hereafter. The initial condition is $A(0, r, \theta)=0$ and the boundary condition is $\left.A\right|_{r \rightarrow \infty}=0$. Note that $\Phi^{i}(\theta)$ are not arbitrary functions and they should be invariant to the choice of direction $\theta=0$ axis. It means $\Phi^{i}(-\theta)=-\Phi^{i}(\theta)$, so $\Phi^{i}=$ const is not possible due to the physics of our problem. For such a case, the convenient replacement is as follows:

$A=\sum_{n=1}^{+\infty} a_{n} p_{n} w_{n}$

where $p_{n} \equiv P_{n}^{1}(\cos \theta)$ are associated Legendre functions which are the solutions of the equation:

$\frac{\partial}{\partial \theta}\left(\frac{1}{\sin \theta} \frac{\partial p_{n} \sin \theta}{\partial \theta}\right)+n(n+1) p_{n}=0$.

They consist of an orthonormal basis $\left(\int_{-1}^{1} p_{n} p_{j} d \cos \theta=0\right.$, if $n \neq j$ and $\left.\int_{-1}^{1} p_{n}^{2} d \cos \theta=2 n(n+1) /(2 n+1)\right)$, so $p_{0}=0$ (Gradshtein and Rizik, 1963, p. 1012). Hence, the coefficients $a_{n}$ can be easily determined:

$a_{n}=\frac{2 n+1}{2 n(n+1)} \int_{-1}^{1} \Phi(\theta) p_{n} d(\cos \theta)$

As a result, $w_{n}$ is a solution of the following equation:

$$
\begin{aligned}
& \frac{1}{D} \frac{\partial w_{n}}{\partial t}-\frac{1}{r^{2}} \frac{\partial}{\partial r}\left(r^{2} \frac{\partial w_{n}}{\partial r}\right)+\frac{n(n+1)}{r^{2}} w_{n} \\
& =B f(t, r) .
\end{aligned}
$$

It is shown in Molchanov et al. (2001) that the solution can be expressed as follows:

$$
\begin{gathered}
w_{n}(t, r)=B \int_{0}^{t} d \tau \int_{0}^{+\infty} \frac{f(\tau, \rho)}{\sqrt{r}} \frac{1}{2 D(t-\tau)} e^{-\frac{r^{2}+\rho^{2}}{4 D(t-\tau)}} \\
I_{n+1 / 2}\left(\frac{r \rho}{2 D(t-\tau)}\right) \rho^{3 / 2} d \rho
\end{gathered}
$$

where $I_{n+1 / 2}(z)$ is the modified Bessel function of the order 

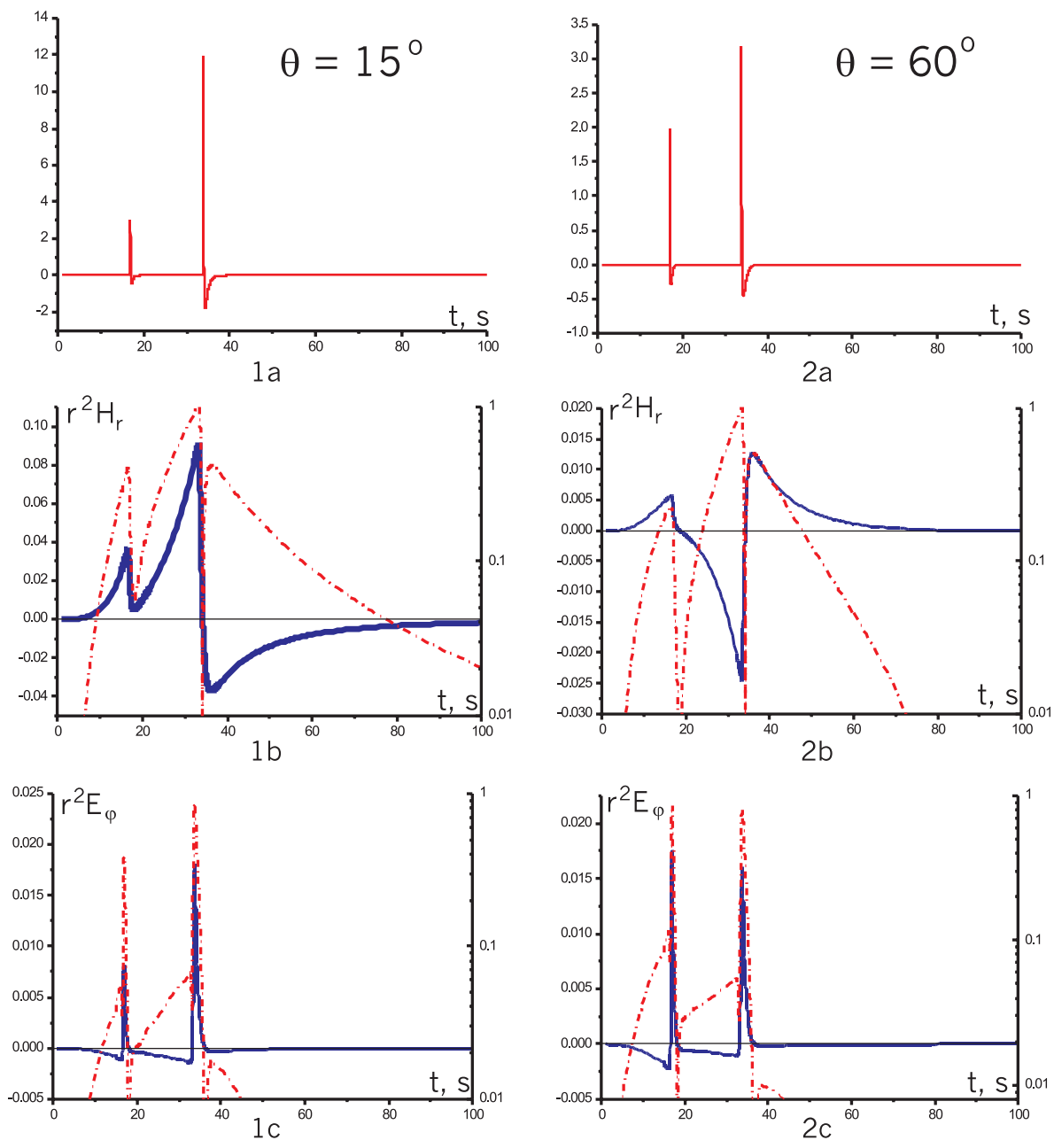

Fig. 5. Seismic source (a), $r^{2} H_{r}$ (b), $r^{2} E_{\varphi}$ (c) as a function of $t ; r=100 \mathrm{~km}, L_{s}=3 \mathrm{~km}, \theta=15^{\circ}(1)$ or $\theta=60^{\circ}(2), C_{p}=6 \mathrm{~km} / \mathrm{s}$, $C_{S}=3 \mathrm{~km} / \mathrm{s}, D=200 \mathrm{~km}^{2} / \mathrm{s}$. Dash-dot lines show the value of $\left|H_{r}\right| / \max \left|H_{r}\right|(2)$ and $\left|E_{\varphi}\right| / \max \left|E_{\varphi}\right|(3)$ in logarithmic coordinates.

of $n+1 / 2$. Note that in the case of $n=0$, it reduces to the well-known Poisson solution of the diffusion equation.

$$
\begin{aligned}
w_{0}(t, r) & =\frac{B}{\pi} \int_{0}^{t} \int_{V_{\rho}} \frac{1}{(4 D(t-\tau))^{3 / 2}} e^{-\frac{|r-\rho|^{2}}{4 D(t-\tau)}} d V_{\rho} d \tau \\
& =\frac{B}{\pi} \int_{0}^{t} d \tau \int_{0}^{\pi} \sin \theta^{\prime} d \theta^{\prime} \int_{0}^{2 \pi} d \varphi^{\prime} \int_{0}^{+\infty} \\
& \frac{1}{(4 D(t-\tau))^{3 / 2}} e^{-\frac{r^{2}+\rho^{2}-2 r \rho\left(\cos \theta \cos \theta^{\prime}+\sin \theta \sin \theta^{\prime} \cos \varphi^{\prime}\right)}{4 D(t-\tau)}} d \rho \\
& =\frac{B}{\sqrt{\pi}} \int_{0}^{t} \frac{d \tau}{\sqrt{D(t-\tau)}} \int_{0}^{+\infty} \\
& f(\tau, \rho) \frac{\rho}{2 r}\left(e^{-\frac{(r-\rho)^{2}}{4 D(t-\tau)}}-e^{-\frac{(r+\rho)^{2}}{4 D(t-\tau)}}\right) d \rho .
\end{aligned}
$$

In our case, $n=1$, and $n=3$. Then we have

$$
\begin{aligned}
& w_{n}(t, r)=\frac{B}{\sqrt{\pi}} \int_{0}^{t} \frac{d \tau}{\sqrt{D(t-\tau)}} \int_{0}^{+\infty} \\
& \left.f(\tau, \rho) F_{n}(D(t-\tau), \rho, r)\right) d \rho, \quad n=1,3
\end{aligned}
$$

where

$$
\begin{gathered}
F_{1}(a, \rho, r)=\frac{\rho}{r} C s(a, \rho, r)-\frac{2 a}{r^{2}} \operatorname{Sn}(a, \rho, r), \\
F_{3}(a, \rho, r)=\left(\frac{\rho}{r}+\frac{60 a^{2}}{\rho r^{3}}\right) \operatorname{Cs}(a, \rho, r) \\
-\left(\frac{12 a}{r^{2}}+\frac{120 a^{3}}{r^{4} \rho^{2}}\right) \operatorname{Sn}(a, \rho, r), \\
C s(a, \rho, r)=\frac{1}{2}\left(e^{-\frac{(r-\rho)^{2}}{4 a}}+e^{-\frac{(r+\rho)^{2}}{4 a}}\right), \\
\operatorname{Sn}(a, \rho, r)=\frac{1}{2}\left(e^{-\frac{(r-\rho)^{2}}{4 a}}-e^{-\frac{(r+\rho)^{2}}{4 a}}\right) .
\end{gathered}
$$

\section{Result of computations}

We use Eq. (21) for an induced source. Let us calculate the coefficients $a_{n}$ for the case of a tension crack:

$\sin \theta\left(1+\cos ^{2} \theta\right)=\frac{6}{5} p_{1}(\cos \theta)+\frac{2}{15} p_{3}(\cos \theta)$, 

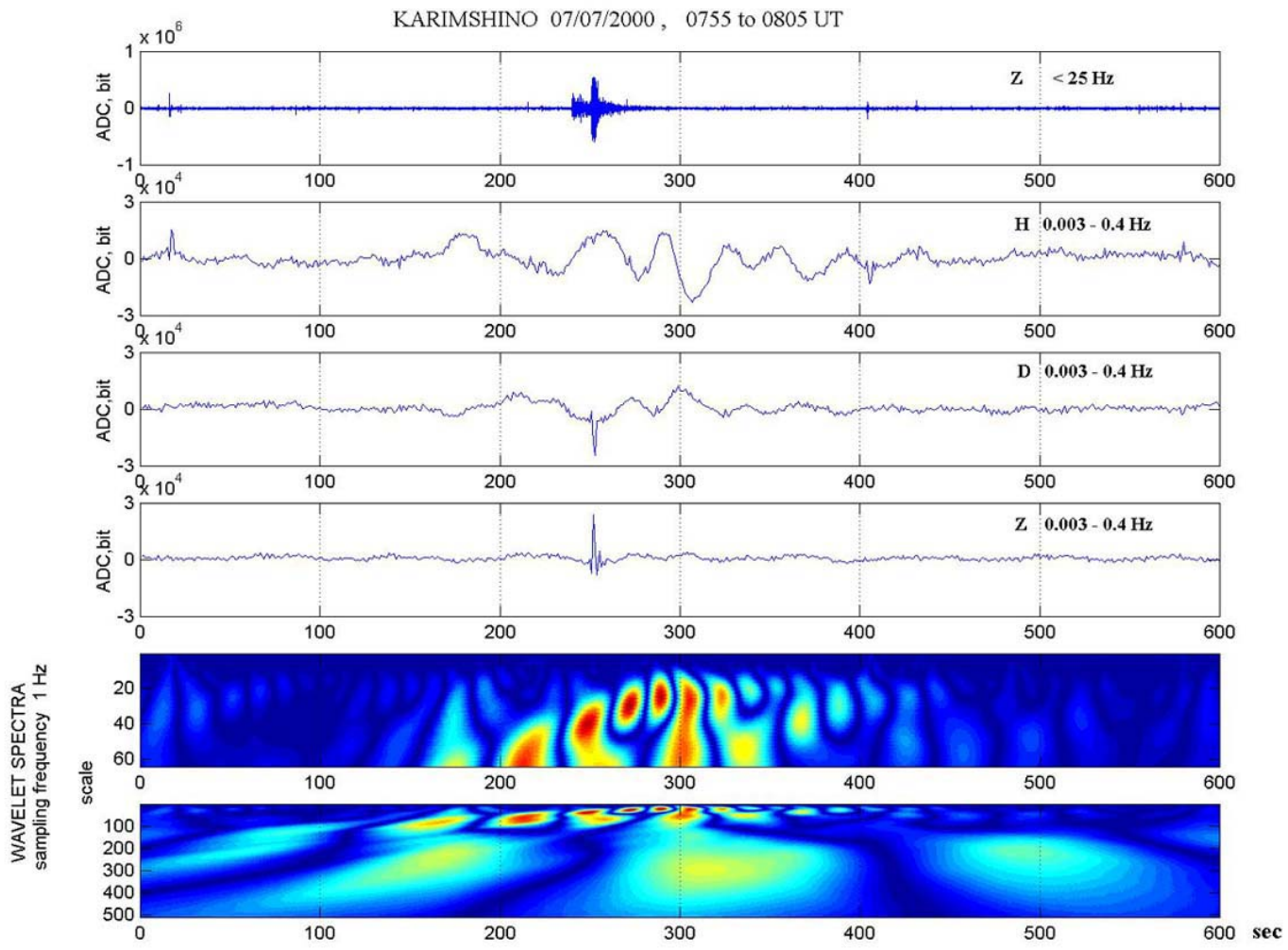

Fig. 6. Seismic pulse (above) together with coseismic variations in the $H, D, Z$ components of the magnetic field $(0.003-0.4 \mathrm{~Hz}$ frequency range, next panels) and wavelet spectrum of $H$-variations (below) observed at Karimshino station, Kamchatka, Russia. Hypocentral distance is $\approx 86 \mathrm{~km}$ and $M_{S}=3.8$ in this case. Influence by shaking in the magnetic field is coincident with the maximal spike inside the seismic train, but the total duration of magnetic pulse $\tau_{m}$ essentially exceeds the length of the seismic pulse $\tau_{s}$ and their ratio $r t=\tau_{m} / \tau_{s} \approx 30$.

$$
\sin \theta \cos ^{2} \theta=\frac{1}{5} p_{1}(\cos \theta)+\frac{2}{15} p_{3}(\cos \theta)
$$

We use Eq. (33) for the calculations when $B=1$. Some results of the calculation are shown in Fig. 4 and Fig. 5. Fig. 4 shows the plots of $r^{2} H_{r}$ as a function of $t$ for $L_{s}=1$ and distances of 50,100 and $150 \mathrm{~km}$. It is clear that the value of $H_{r}$ decreases as $\sim 1 / r^{2}$.

Plots of the source (1a, 1b), $\operatorname{Hr}(2 \mathrm{a}, 2 \mathrm{~b})$ and $E_{\varphi}(3 \mathrm{a}, 3 \mathrm{~b})$ for $\theta=15^{\circ}, \theta=60^{\circ}$ are shown in Fig. 5. To define an elongation of the magnetic field and electric field, we placed the graphics of $\left|H_{r}\right| / \max \left|H_{r}\right|$ and $\left|E_{\varphi}\right| / \max \left|E_{\varphi}\right|$ in logarithmic coordinates (dash-dot line).

\section{Discussion and conclusions}

We have tried to demonstrate that consideration of the penetration of a low-frequency wave through a conductive medium is rather simplified if we solve the equations of electromagnetic diffusion by a double-potential method. This approach is related to the specific gauge condition, which was justified in detail here. Of course, the solutions are not so simple when taking into consideration inhomogeneity and the presence of boundaries at the real medium. But even in this situation, the discussed solutions could be useful for estimates (assuming some averaged parameters) or for computations in the case of an inhomogeneous medium.

The most prominent feature of the seismo-inductive effect is the elongation of magnetic and electric pulses in comparison with the duration of the seismic pulse, as demonstrated in Fig. 5. Nagao et al. (2000) found that duration of coseismic electric pulses are sometimes exceeding essentually the length of the corresponding seismic pulses. They interpreted this in terms of electro-kinetic phenomenon, but a seismoinductive effect explanation is also possible. Recently, some indication of coseismic magnetic pulses have probably been found in Kamchatka ULF observations (description of the observations is presented in Gladyshev et al., 2001). An example is shown in Fig. 6.

Of course, there is no exact coincidence in theoretical and observational results; however, a tendency of magnetic pulse elongation is evident from both pictures in Fig. 5 and Fig. 6.

It is known that a variety of seismic shocks could happen in a time of foreshock activity during several days before a large earthquake. The usual seismic spectrum has a maximum near $\omega / 2 \pi \sim \omega_{c} / 2 \pi \sim 1 \mathrm{~Hz}$ (see e.g. Molchanov et 
al., 2001). Let us imagine what will happen with the magnetic and electric variations, taking into account a possibility of the inductive effect discussed here. We can expect an appearance of specific electromagnetic "noise" whose spectrum maximum is reduced down to $\omega / 2 \pi \sim 1 \mathrm{~Hz} / r t \sim$ $0.01-0.05 \mathrm{~Hz}$. Such phenomena in the shown frequency range have been observed indeed as ULF electromagnetic emission and they are well discussed just in this monograph. We can expect, furthermore, that this ULF emission (if resulted from an inductive effect) should have the same limitation in the area of observation as foreshock seismic shocks thenselves. In the case of EQ with $M_{s} \sim 5-6$, the usual values of foreshock magnitudes which occurr near the hypocentral region are about 3-4 $\left(L_{s} \sim 1-2 \mathrm{~km}\right)$ and the attenuation distance for them is $R_{a} \approx Q L_{s} \sim 100-200 \mathrm{~km}$. Similar limiting distance in the observation of precursory ULF magnetic emission was often reported. In fact, this limitation is more severe for electromagnetic pulses due to the $\sim 1 / r^{2}$ dependence of their amplitudes even in the region $\left(r \leq R_{a}\right)$ without attenuation as evident from Fig. 5 and unlike the geometrical factor $1 / r$ for the seismic pulses.

Our modeling is greatly simplified due to the suppositions of an inhomogeneous medium, the special form of the fracture (in reality, shear crack is more probable) and the orientation of the fracture plane to the direction of the external magnetic field. It is interesting to note that some important features of the seismo-inductive effect are revealed even in this oversimplified consideration.

\section{References}

Aki, K. and Richards, P. G.: Quantitative seismology, 1980.

Belov, S. V., Migunov, N. I., and Sobolev G. A.: Magnetic effects caused by great earthquakes in Kamchatka, Geomagnetism and Aeronomy, 14, 2, 380-382, (in Russian), 1974.

Brune, J. N., Archuleta, R. J., and Hartsell, S.: Far-field S-wave spectrum, corner frequency and pulse shapes, J. Geophys. Res., 84, 2262-2272, 1979.

Eleman, F.: The response of magnetic instruments to earthquake waves, J. Geomagn. Geoelectr., 18, 1, 43-72, 1965.

Fenoglio, M. A., Johnston, M. J. S., and Byerlee, J. D.: Magnetic and electric fields associated with changes in high pore pressure in fault zone-application to the Loma Prieta ULF emissions, Proc. of Workshop LXIII, Menlo Park, CA, 262-278, 1994.

Gladyshev, V., Baransky, L., Schekotov, A., Fedorov, E., Pokhotelov, O., Andreevsky, S., Rozhnoi, A., Khabazin, Y., Belyaev, G., Gorbatikov, A., Gordeev, E., Chebrov, V., Sinitsin, V., Lutikov, A., Yunga, S., Kosarev, G., Surkov, V., Molchanov, O., Hayakawa, M., Uyeda, S., Nagao, T., Hattori, K., and Noda, Y.: Some preliminary results of seismo-electromagnetic research at Complex Geophysical Observatory, Kamchatka in: the book "Seismo Electromagnetics (Lithosphere-AtmosphereIonosphere Coupling)" (Eds) Hayakawa, M. and Molchanov, O .A., TERRAPUB, Dec., 2001.

Gradshtein, I. S. and Rizhik, I. M.: Tables of integrals sums and rows, Izd. Phys.-Math Literature, Moscow, 1963.

Hayakawa, M.: (Ed), Atmospheric and Ionospheric Electromagnetic Phenomena Associated with Earthquakes, Terra Sci. Pub. Comp., Tokyo, pp. 996, 1999.

Hohmann, G. W.: Numerical modeling for electromagnetic methods of geophysics, in Electromagnetic methods in applied geophysics, v.1, Theory, (Ed) Nabighian, M. N., 314-364, 1988.

Kawate, R., Molchanov, O. A., and Hayakawa, M.: Ultra-lowfrequency magnetic fields during the Guam earthquake of $8 \mathrm{Au}-$ gust 1993 and their interpretation, Phys. Earth Planet. Inter., 105, 229-238, 1998.

Landau, L. and Liftshits, E.: Electrodynamics of the continuous medium, Nauka, Moscow, pp. 661, 1992.

Molchanov, O. A.: "Fracturing as an underlying mechanism of seismo-electric signals", in: Atmospheric and Ionospheric Electromagnetic Phenomena associated with Earthquakes, (Ed) Hayakawa, M., Terra Scientific Pub. Company, Tokyo, Japan, 1999.

Molchanov, O. A. and Hayakawa, M.: Generation of ULF electromagnetic emissions by microfracturing, Geophys. Res. Lett., 22, 22, 3091-3094, 1995.

Molchanov, O. A., Kulchitsky, A. V., and Hayakawa, M.: ULF emission due to inductive seismo-electromagnetic effect, in: book "Seismo Electromagnetics (Lithosphere-AtmosphereIonosphere Coupling)", (Eds) Hayakawa, M. and Molchanov, O .A., TERRAPUB, Dec., 2001.

Nagao, T., Ogihara, Y., Yamaguchi, T., Takahashi, I., Hattori, K., Noda, Y., Sayanagi, K., and Uyeda, S.: Co-seismic geoelectric potential changes observed in Japan, Geophys. Res. Lett., 27, 1535-1538, 2000.

Pellerin, L. and Hohmann, G. W.: A parametric study of the vertical electric source, Geophysics, 60, 43-52, 1995.

Scholz, C. H.: The mechanics of earthquakes and faulting, Cambridge Univ. Press, 439 pp., 1990.

Surkov, V. V.: ULF electromagnetic perturbations resulting from the fracture and dilatancy in the earthquake preparation zone, in: "Atmospheric and Ionospheric Phenomena Associated with Earthquakes", (Ed) Hayakawa, M., Terra Sci. Pub Comp., Tokyo, p. 357-370, 1999.

Surkov, V. V., Molchanov, O. A., and Hayakawa, M.: Preearthquake ULF electromagnetic emission as result of inductive seismo-magnetic phenomena during microfracturing, submitted to J. Atm. Solar Terr. Phys., 2000.

Takeuchi, N., Chubachi, N., and Narita, K.: Observation of earthquake waves by the vertical earth potential difference method, Phys. Earth Planet. Inter., 101, 157-161, 1997.

Ward, S. H. and Hohmann, G. W.: Electromagnetic theory for geophysical applications, in Electromagnetic methods in applied geophysics, v.1, Theory, (Ed) Nabighian, M. N., 131-313, 1988. 